\section{Unusual Extensive T1 Hyperintense Signals on MR Imaging in Neurocysticercosis}

Neurocysticercosis (NCC) is a common cause of morbidity in many developing countries and a common cause of seizure disorder in children and adults. The 4 stages of NCC are vesicular, vesicular colloidal, granular nodular, and nodular calcified. ${ }^{1}$ The appearance on MR imaging would depend on the stage of the disease. Most commonly, NCC presents as isohypointense lesions on T1-weighted MR imaging. To our knowledge, extensive hyperintense signals on T1-weighted images have not been described for NCC.

A young man 22 years of age presented with headache on and off for 5-6 months. There was no history of seizures, which was also very unusual in his case. Plain and contrast MR imaging of the brain was performed. T1-weighted images revealed multiple hyperintense foci of varying sizes, in bilateral cerebral, cerebellar hemispheres and the brain stem (Fig 1). The T1 hyperintense signals were thicker in the right cerebellar hemisphere (Fig 2). Multiple other lesions were revealed on gradient images, which were not demonstrated on routine MR images. These multiple extensive T1 hyperintense signals were confirmed to be calcifications on CT, which was performed subsequent to the MR imaging. Few of the T1 hyperintense lesions showed mild enhancement on contrast administration.

The MR imaging appearance and contrast characteristics would depend on the stage of the parasitic cyst. The unusual appearance of such extensive T1 hyperintense signals, as seen in our patient, has not been described earlier and is probably due to calcifications, which are known to appear hyperintense on T1-weighted MR imaging. ${ }^{2,3}$ The reason for calcifications demonstrating $\mathrm{T} 1$ hyperintense signals has not been clearly elucidated. ${ }^{2}$ These signals in calcifications are probably due to shortening of the T1 relaxation time, due to the paramagnetic effect of calcium on adjacent water. ${ }^{2}$ The degree of $\mathrm{T} 1$ shortening is larger with a larger area of calcification. Calcifications may also appear hyperintense on the T1-weighted images if they contain other paramagnetic elements like manganese or iron. ${ }^{2}$ Such extensive T1 hyperintense calcifications with blooming on gradient images must not be mistaken for multiple bleeds.

\section{References}

1. Osborne AG. Diagnostic Neuroradiology: The Requisites. St Louis: Mosby; 2003:709-12

2. Tawil MI, Wilson JP, Wright NB. Intracranial lithography? $\mathrm{Br} J$ Radiol 2002:75:563-64

3. Dell LA, Brown MS, Orrison WW, et al. Physiologic intracranial calcification with hyperintensity on MR imaging: case report and experimental model. AJNR Am J Neuroradiol 1988;9:1145-48

P. Lalitha

B. Reddy

Department of Radiology Focus Diagnostics Hyderabad, India

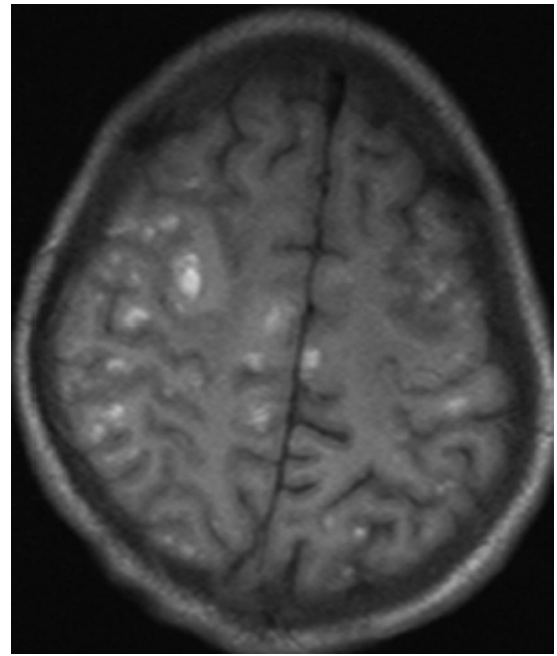

Fig 1. Plain T1-weighted image reveals small hyperintense foci in the bilateral cerebra hemispheres.

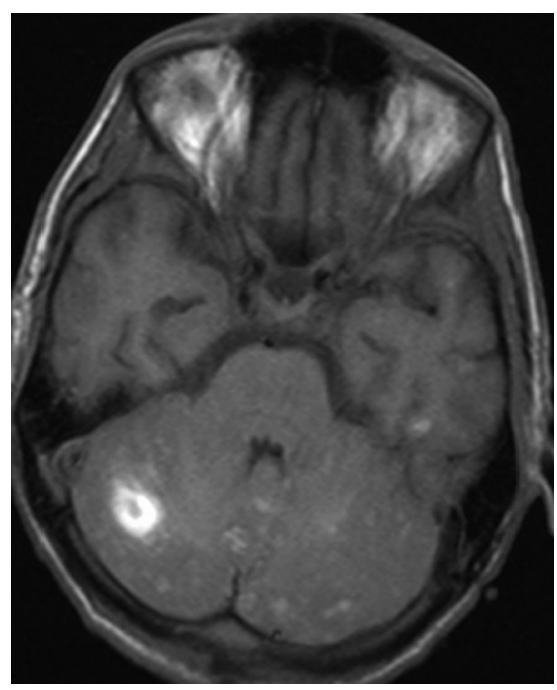

Fig 2. Plain T1-weighted image reveals hyperintense signals in both cerebellar hemispheres, thicker on the right side. 